\title{
Aspirin reduces lipopolysaccharide-induced pulmonary inflammation in human models of ARDS
}

\author{
U Hamid, ${ }^{1}$ A Krasnodembskaya, ${ }^{1}$ M Fitzgerald, ${ }^{1}$ M Shyamsundar, ${ }^{1}$ A Kissenpfennig, ${ }^{1}$ \\ C Scott, ${ }^{2}$ E Lefrancais, ${ }^{3} \mathrm{M}$ R Looney, ${ }^{3}$ R Verghis, ${ }^{4}$ J Scott, $^{5}$ A J Simpson, ${ }^{5}$ \\ J McNamee, ${ }^{6}$ D F McAuley, ${ }^{1,6}$ C M O'Kane ${ }^{1}$
}

- Additional material is published online only. To view please visit the journal online (http://dx.doi.org/10.1136/ thoraxjnl-2016-208571).

For numbered affiliations see end of article.

Correspondence to Dr Cecilia O'Kane, Centre for Experimental Medicine, School of Medicine, Dentistry and Biomedical Sciences, Queen's University Belfast, Wellcome Wolfson Building, 97 Lisburn Road, Belfast, Northern Ireland BT9 7AE, UK;

c.okane@qub.ac.uk

Received 1 March 2016 Revised 24 November 2016 Accepted 3 December 2016 Published Online First 12 January 2017

\section{CrossMark}

To cite: Hamid $U$, Krasnodembskaya A, Fitzgerald M, et al. Thorax 2017;72:971-980.

\section{ABSTRACT}

Rationale Platelets play an active role in the pathogenesis of acute respiratory distress syndrome (ARDS). Animal and observational studies have shown aspirin's antiplatelet and immunomodulatory effects may be beneficial in ARDS.

Objective To test the hypothesis that aspirin reduces inflammation in clinically relevant human models that recapitulate pathophysiological mechanisms implicated in the development of ARDS.

Methods Healthy volunteers were randomised to receive placebo or aspirin 75 or $1200 \mathrm{mg}(1: 1: 1)$ for seven days prior to lipopolysaccharide (LPS) inhalation, in a double-blind, placebo-controlled, allocation-concealed study. Bronchoalveolar lavage (BAL) was performed 6 hours after inhaling $50 \mu \mathrm{g}$ of LPS. The primary outcome measure was BAL IL-8. Secondary outcome measures included markers of alveolar inflammation (BAL neutrophils, cytokines, neutrophil proteases), alveolar epithelial cell injury, systemic inflammation (neutrophils and plasma C-reactive protein (CRP)) and platelet activation (thromboxane B2, TXB2). Human lungs, perfused and ventilated ex vivo (EVLP) were randomised to placebo or $24 \mathrm{mg}$ aspirin and injured with LPS. BAL was carried out 4 hours later. Inflammation was assessed by BAL differential cell counts and histological changes.

Results In the healthy volunteer $(n=33)$ model, data for the aspirin groups were combined. Aspirin did not reduce $B A L I L-8$. However, aspirin reduced pulmonary neutrophilia and tissue damaging neutrophil proteases (Matrix Metalloproteinase (MMP)-8/-9), reduced BAL concentrations of tumour necrosis factor $\alpha$ and reduced systemic and pulmonary TXB2. There was no difference between high-dose and low-dose aspirin. In the EVLP model, aspirin reduced BAL neutrophilia and alveolar injury as measured by histological damage.

Conclusions These are the first prospective human data indicating that aspirin inhibits pulmonary neutrophilic inflammation, at both low and high doses. Further clinical studies are indicated to assess the role of aspirin in the prevention and treatment of ARDS.

Trial registration number NCT01659307 Results.

\section{INTRODUCTION}

The acute respiratory distress syndrome (ARDS) is characterised by an uncontrolled alveolar inflammatory response, the hallmarks of which are neutrophil infiltration, protein-rich pulmonary oedema

\section{Key messages}

What is the key question?

- Experimental animal and human observational studies suggest that aspirin may be beneficial in acute respiratory distress syndrome (ARDS): however, whether aspirin can reduce inflammation and injury in the human alveolus has not been assessed prospectively.

\section{What is the bottom line?}

- In two human models of ARDS, we show that aspirin reduces pulmonary neutrophil infiltration and alveolar inflammation and injury.

\section{Why read on?}

- Aspirin is effective in reducing neutrophil-mediated inflammation in the human alveolus: these findings support further clinical trial testing of aspirin to treat neutrophil-driven pulmonary diseases including ARDS.

- Additional data and information are available in the online supplementary data.

accumulation, hypoxia and the need for mechanical ventilation. Platelets may contribute to the pathophysiology of ARDS. Platelet activation, degranulation and aggregation within the lung can lead to further vasodilatation, with chemoattraction of neutrophils and monocytes, increasing coagulation and the deposition of a provisional matrix, and inducing fibroblast activation and proliferation, all features of ARDS. ${ }^{12}$

Additionally, the formation of neutrophil or monocyte platelet aggregates can drive inflammation: neutrophils seek out activated platelets within the circulation and interact with them via PSGL-1-a glycoprotein ligand for P-selectin-to initiate inflammation. ${ }^{3}$ This process is implicated directly in several in vivo models of acute lung injury, including acid-induced Acute Lung Injury (ALI) and lipopolysaccharide (LPS)-induced injury. ${ }^{4}$

Recently, platelets have also been shown to play an important role in the formation of neutrophil-extracellular traps (NETs). ${ }^{6}$ NETs formation allows activated neutrophils to release chromatin material interlaced with granular proteins 
and proteases, which traps pathogens but also damages endothelial cells and drives neutrophil recruitment to the lungs. ${ }^{7}$ An increase in NETs has been detected in plasma of patients with transfusion-related $\mathrm{ARDS}^{7}$ and is also implicated in the pathophysiology of ARDS of other aetiologies. ${ }^{89}$

Aspirin is widely used as a highly effective antiplatelet in the primary and secondary prevention of cerebrovascular, coronary artery and peripheral vascular disease. In addition to its antiplatelet effects, it increases the production of resolvins and lipoxins, which may reduce inflammation and promote resolution. ${ }^{10}$ Various experimental studies of ARDS have shown a beneficial effect of aspirin on reducing pulmonary inflammation. ${ }^{11}$ Similarly, in observational studies, patients on aspirin therapy prior to hospitalisation had a reduced incidence of ARDS $^{12} 13$ and organ dysfunction ${ }^{14}$. In contrast, the recent LIPS-A study, which prospectively treated patients at risk of developing ARDS with aspirin, showed no reduction in incidence of ARDS. ${ }^{15}$ The study had limitations, with a lower incidence of ARDS than expected, and the population was much less ill, with a lower incidence of mechanical ventilation, acute kidney injury and mortality, than predicted. As such the study was underpowered for the primary and secondary endpoints. In addition, over half the screened potentially eligible population was excluded due to preexisting antiplatelet use or pre-existing bilateral infiltrates. The authors acknowledged that the study was underpowered and concluded the exclusion of large cohorts significantly limited the generalisability of the findings. In a single-centre observational study, we found use of aspirin (at antiplatelet doses) in patients with ARDS either prior to or during their Intensive Care Unit (ICU) stay was associated with a reduction in ICU mortality. ${ }^{16}$

Most animal studies have used a higher dose of aspirin, while observational studies have reported a beneficial effect with $75-81 \mathrm{mg}$ of aspirin. ${ }^{12}$ There have been no prospective experimental studies testing whether aspirin can directly affect inflammation within the human lung (LIPS-A addressed only markers of systemic inflammation ${ }^{15}$ ) or whether high or low doses are necessary to attenuate pulmonary inflammation in humans.

The objective of this study was to test if aspirin can reduce pulmonary inflammation in two human models of ARDS and to identify if high-dose aspirin was more effective than low dose in reducing pulmonary inflammation.

Inhalation of LPS has been previously shown to produce pulmonary inflammation, which is qualitatively representative of ARDS without any associated serious adverse outcomes, ${ }^{17}$ and has been used to test anti-inflammatory or pro-repair interventions to inform clinical trials for the prevention or treatment of ARDS. ${ }^{17}$ The model of injuring isolated perfused and ventilated human lungs ex vivo allows the testing of anti-inflammatory therapeutics in a human setting, with the advantages of being able to use a more injurious stimulus than in the healthy volunteerinhaled LPS model, and allowing histological examination. ${ }^{18-20}$ Instilling LPS in this ex vivo lung perfusion (EVLP) model drives neutrophilic pulmonary inflammation, impaired fluid clearance and elevation in bronchoalveolar lavage (BAL) concentrations of the pro-inflammatory cytokines that characterise ARDS. ${ }^{18}$

In this study, we show that aspirin reduces neutrophilic pulmonary inflammation and that low-dose and high-dose aspirin have similar effects within the pulmonary compartment.

\section{METHODS}

\section{In vivo model of pulmonary inflammation in healthy} volunteers

The study, the effect of aspirin on reducing inflammation in a human in vivo model of acute lung injury (ARENA), was registered at ClinicalTrials.gov (NCT01659307) and was approved by the local research ethics committee (ORECNI).

\section{Volunteers}

Thirty-five healthy non-smoking subjects, taking no regular medication, recruited by advertising, underwent screening assessment (history, physical examination, routine blood investigation, ECG and spirometry).

Exclusion criteria were age $<18$ years, pregnancy, breast feeding or female of childbearing potential not taking adequate contraception, participation in a clinical trial of an investigational medicinal product within 30 days, aspirin or non-steroidal anti-inflammatory use in the past four weeks, history of asthma, known aspirin or non-steroidal anti-inflammatory drug hypersensitivity, history of peptic ulcer disease, platelet count $<150 \times 10^{6} / \mathrm{mL}$, known aspirin resistance.

The study was carried out in the Wellcome Trust-Wolfson Northern Ireland Clinical Research Facility at Belfast City Hospital.

\section{Study design}

This was a randomised, double-blinded, placebo-controlled allocation-concealed clinical study in which subjects were randomised to aspirin $1200 \mathrm{mg}, 75 \mathrm{mg}$ or placebo $(1: 1: 1)$. Randomisation was undertaken by an independent clinical trials pharmacist using the statistical software nQuery Advisor. Mixed block sizes (of 3, 6 and 9) and no stratification were used. Blinding was achieved by encapsulation with gelatin. The aspirin capsule contained the aspirin tablet with lactose powder. The placebo capsule contained lactose powder only. Both aspirin and placebo study drugs had an identical appearance. Subjects took the study medication for 7 days before inhalation of LPS. On day 7,30 min after the study medicine was taken under direct observation by the study team, subjects inhaled $50 \mu \mathrm{g}$ LPS (O26:B6, Sigma) using an automated nebulizer (Spira dosimeter, Finland) as previously described. ${ }^{17}$

Blood was collected immediately before the subject inhaled the LPS and also at 6 and 24 hours after LPS inhalation. BAL was performed 6 hours after LPS inhalation, at which point we have previously identified a robust neutrophilic inflammatory response. Processing of BAL fluid (BALF) and blood was performed as described previously. ${ }^{17}{ }^{21}$ The study schedule is summarised in online supplementary figure S1 in the appendix.

The primary outcome of the study was BAL interleukin (IL)-8 concentration. The sample size was calculated as 11 per group to detect a $30 \%$ change in BAL IL- 8 , with $80 \%$ power at twotailed significance of $<0.05$, with aspirin. This was based on a previous study where BAL IL-8 concentration after LPS inhalation by healthy volunteers was $389 \pm 94 \mathrm{pg} / \mathrm{mL},{ }^{17}$ and evidence that aspirin reduced systemic IL-8 by up to $30 \%$ in a cohort of patients undergoing dialysis. ${ }^{22}$ The main secondary efficacy outcomes were markers of alveolar inflammatory response (BAL neutrophil counts, BAL inflammatory cytokines), alveolar epithelial injury (albumin, protein permeability ratio, SP-D and MMP-7) and plasma inflammatory response as assessed by CRP 24 hours after LPS inhalation.

\section{EVLP model}

This model was adapted from the methods described by Lee et $a .^{18}$ Briefly, lungs obtained from the International Institute for the Advancement of Medicine, with consent for use in research, were perfused, rewarmed with Dulbecco's Modified Eagle Medium (DMEM) with L-glutamine and 5\% albumin (perfusate) and inflated with $10 \mathrm{~cm} \mathrm{H}_{2} \mathrm{O}$ CPAP. BAL was carried 
out at baseline once a temperature of $35^{\circ} \mathrm{C}$ was reached. Whole blood $(100 \mathrm{~mL})$ obtained from a healthy volunteer was incubated for 1 hour with $24 \mathrm{mg}$ aspirin and added to the perfusate (giving a final concentration of aspirin of $21 \mu \mathrm{g} / \mathrm{mL}$ in the perfusate, which corresponds to peak plasma levels obtained after a $\left.300 \mathrm{mg} \mathrm{dos} \mathrm{e}^{23}\right)$. Simultaneously LPS $(6 \mathrm{mg}$ ) was introduced into a preselected lobe. At 4 hours, BAL was repeated. Experiments were carried out for six lungs in the placebo group and five lungs in the aspirin-treated group.

The use of human lungs and blood was approved by Queen's University of Belfast School of Medicine, Dentistry and Biomedical Sciences Research Ethics Committee. The study schedule is shown in online supplementary figure S2. A detailed description of the EVLP model is included in the online supplementary data.

The investigator carrying out the laboratory analyses was kept blinded to the treatment assignation group of the normal volunteers or the ex vivo lungs throughout.

\section{BAL cytokines and proteases}

These were measured using cytometric bead array or ELISA as previously described ${ }^{17}$ and are detailed in the online supplementary data.

\section{BAL protein and permeability measurements}

Total protein was measured by Bradford assay. BAL albumin was measured by an immunoturbidimetric method by Randox Testing Services, UK. IgG was measured by ELISA (Immundiagnostik). Protein permeability ratio was expressed as ratio of BAL IgG:total protein.

\section{Neutrophil extracellular traps}

To quantify NETs in human plasma, a capture ELISA based on Neutrophil Elastase (NE) associated with DNA was used. ${ }^{9}$ A detailed description is provided in the online supplementary data.

\section{Leucocyte platelet aggregates}

Neutrophil-platelet and monocyte-platelet aggregates were measured in blood at baseline and 6 hours after LPS inhalation as previously described. ${ }^{5}$ A detailed description is provided in the online supplementary data.

\section{Phagocytosis assay}

Neutrophils isolated from peripheral blood were allowed to adhere to tissue culture plastic for $30 \mathrm{~min}$ prior to treatment with aspirin $(0.1$ or $0.5 \mathrm{mM})$. Cells were then exposed to autologous serum-opsonised zymosan for $30 \mathrm{~min}$, washed with PBS, air dried, fixed with methanol and stained with Giemsa to determine the percentage of neutrophils containing two or more zymosan particles. ${ }^{24}$ Further details are given in the online supplementary data.

\section{Lung injury severity score}

Lung sections from the injured lobes in the EVLP model were examined histologically. Details of the scoring system ${ }^{25}$ are given in the online supplementary data. The scorer was blinded to the group allocation.

\section{Statistical analysis}

In the healthy volunteer study, the a priori plan was to present and compare data from the combined aspirin-treated groups with placebo. A secondary analysis to determine if results in the high-dose and low-dose groups differed was carried out. There were no planned interim analyses. Aspirin and placebo groups were compared by Mann-Whitney U (MWU) test. Data for the EVLP study were analysed using GraphPad Prism (GraphPad Software; San Diego, California, USA). A p value of $\leq 0.05$ was considered significant.

\section{RESULTS}

Thirty-five healthy subjects were randomised; two withdrew before progressing to LPS inhalation and bronchoscopy (one decided not to proceed with the study, and one developed an upper respiratory tract infection (URTI), so LPS inhalation and bronchoscopy were considered inappropriate). Randomisation continued until 33 volunteers completed the study protocol. There were 13 in the placebo cohort and 10 in each aspirin cohort ( $\mathrm{n}=20$ aspirin in total) (figure 1). Baseline parameters including age, sex, body mass index or lung volumes are shown in table 1.

Aspirin was well tolerated with no increased incidence of adverse effects in the aspirin-treated cohort. There were no significant changes in $\mathrm{FEV}_{1}$, FVC, oxygen saturation measured by pulse oximetry or vital signs over the course of the study. There were no serious adverse events or suspected unexpected serious adverse reactions to trigger an unscheduled data monitoring committee meeting. A detailed description of the monitoring and adverse events (see online supplementary table S1, ST1) experienced by the volunteers is included in the online supplementary data.

\section{Aspirin reduces BAL neutrophilia but not BAL IL-8}

Aspirin pretreatment did not significantly reduce the prespecified primary outcome measure IL-8 (figure 2A). However, aspirin reduced neutrophil count in the BAL fluid of healthy volunteers who inhaled LPS (figure $2 \mathrm{~B}$ ). There was no significant change in BAL macrophage or lymphocyte counts (table 2), nor BAL NETs-data not shown.

\section{Aspirin reduces neutrophil-derived enzyme secretion in the alveolar space}

Having established that aspirin reduces BAL neutrophilia in the human lung in response to LPS, we investigated the effect on markers of neutrophil activity. The neutrophil-specific enzyme MMP-8 was significantly reduced in the aspirin-treated group (figure 3A). MMP-9, also secreted by activated neutrophils (and other cells), was significantly reduced in the aspirin-treated group (figure 3B). The major secreted inhibitors of these MMPs, Tissue Inhibitors of Metalloproteinase (TIMPs)-1/-2, were unaffected by aspirin treatment (see online supplementary figure S3a and S3b). Both myeloperoxidase (MPO) and neutrophil elastase $(\mathrm{NE})$ showed a trend to reduction in response to aspirin (figure 3C, D) but this did not reach statistical significance.

\section{Aspirin reduces BAL TNF $\alpha$ in healthy volunteers who have inhaled LPS}

To investigate the effect of aspirin on the inflammatory milieu of the LPS-injured alveolus, we measured key cytokines that drive injury in ARDS. Aspirin reduced BAL concentrations of the inflammatory cytokine $\mathrm{TNF} \alpha$ (table 3). There was a non-statistically significant trend towards reduction in all other measured pro-inflammatory cytokines, particularly IL-6, $p=0.07$ (table 3). The IL-1ß:IL-1Ra ratio, a functional marker of unopposed IL-1 $\beta$ activity was reduced but again did not reach statistical significance (table 3). Aspirin had no effect on BAL CRP. 


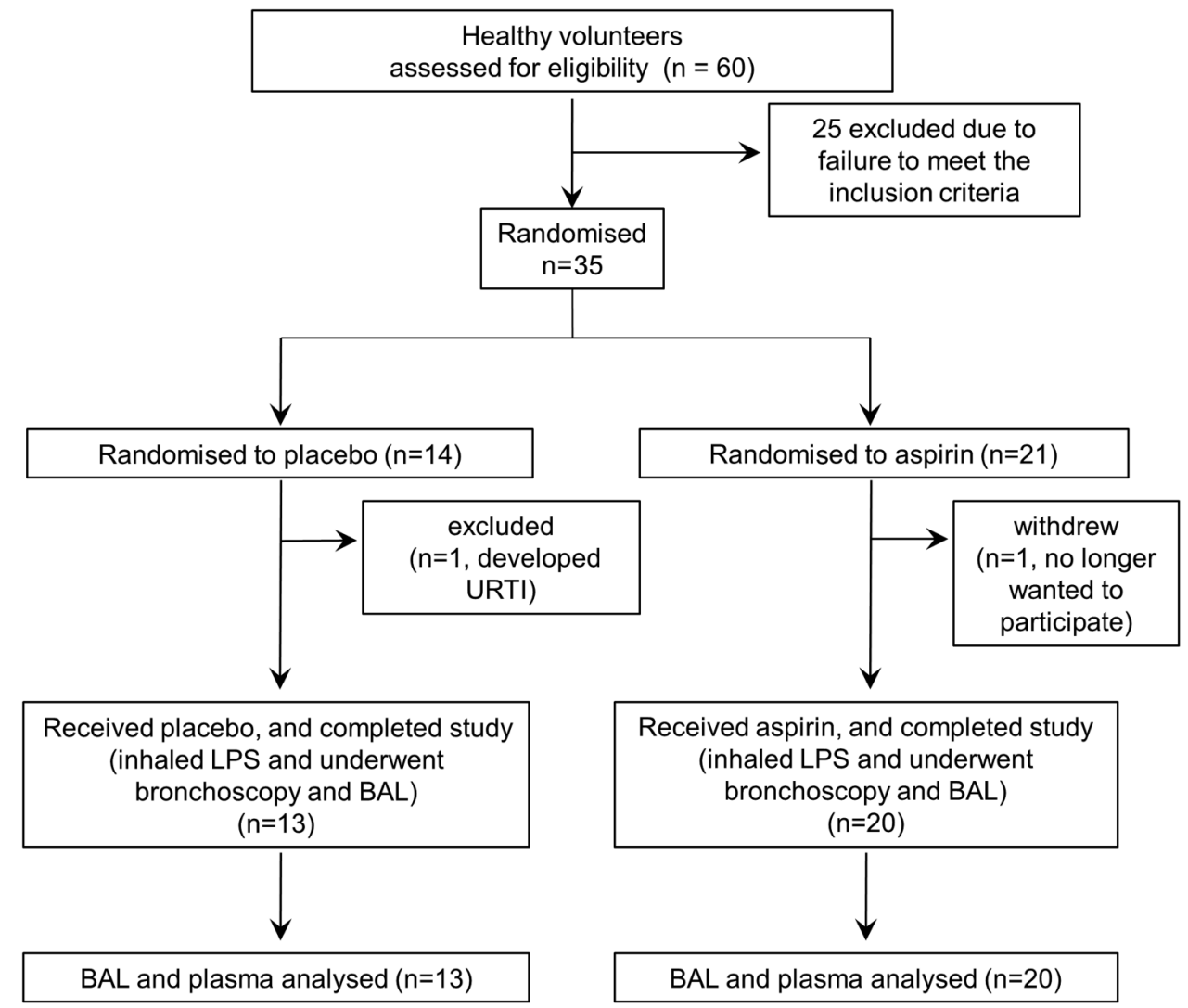

Figure 1 CONSORT diagram of healthy volunteer trial. BAL, bronchoalveolar lavage; LPS, lipopolysaccharide.

\section{Aspirin did not affect epithelial barrier function in response to LPS}

Aspirin had no effect on epithelial barrier function as measured by BAL total protein (not shown), albumin (figure 4A) or IgG: total protein ratio (figure $4 \mathrm{~B}$ ). Interestingly, aspirin also had no effect on secretion of the type II epithelial specific marker SP-D (figure 4C), nor on MMP-7, an epithelial-derived protease in the alveolus (figure 4D).

\section{The effect of aspirin on systemic inflammatory response}

While there was a trend towards a reduction in peripheral neutrophil count, and in peripheral NET formation in the aspirin-treated group 6 hours after LPS inhalation these did not reach significance (figure $5 \mathrm{~A}, \mathrm{p}=0.068$, and figure $5 \mathrm{~B}, \mathrm{p}=0.086$ ). Aspirin did not reduce peripheral CRP (see online supplementary data figure S4), nor peripheral leucocyte platelet aggregate (LPA) formation (see online supplementary figure S5). Importantly when human primary neutrophils were treated with aspirin in vitro,

Table 1 Baseline characteristics of healthy volunteers inhaling lipopolysaccharide

\begin{tabular}{lll}
\hline Characteristics & Placebo & Aspirin \\
\hline Age & $24 \pm 4$ & $27 \pm 10$ \\
Sex (\% female) & 35 & 53 \\
Body mass index & $23 \pm 3$ & $25 \pm 3$ \\
FEV $($ L) & $3.8 \pm 1$ & $4.1 \pm 1$ \\
FVC (L) & $4.4 \pm 1$ & $4.9 \pm 1$ \\
\hline
\end{tabular}

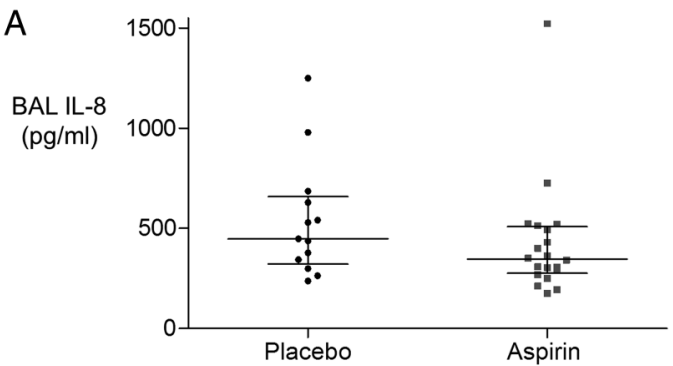

B

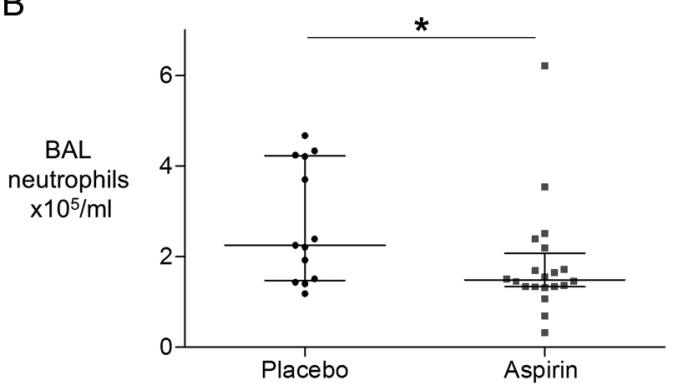

Figure 2 Aspirin does not significantly reduce bronchoalveolar lavage (BAL) interleukin (IL)-8 in the healthy volunteer model of lipopolysaccharide (LPS)-induced lung injury, but does reduce neutrophil recruitment to the alveolar space. Healthy human subjects were randomised to placebo $(n=13)$ or aspirin $(n=20)$ for 7 days prior to LPS inhalation $(50 \mu \mathrm{g})$ and underwent BAL 6 hours later. (A) IL-8 was measured in BAL fluid, but was not significantly reduced, $p=0.11$ (B) Neutrophils in BAL fluid were counted on cytospin and were reduced by over a third in the aspirin-treated group (estimated difference in median $=0.8 \times 10^{5} / \mathrm{mL}, 95 \%$ Cls 0.06 to $2.1 ;{ }^{*} p=0.03$ for aspirin vs placebo). 
Table 2 BAL Differential Cell Count

\begin{tabular}{llrr}
\hline Bronchoalveolar lavage & Placebo & Aspirin & Difference in median (95\% Cls) \\
\hline Macrophages $(\times 105 / \mathrm{mL})$ & $2.99(2.30-4.24)$ & $2.96(1.44-3.71)$ & $0.4(-0.71$ to 1.49$)$ \\
Lymphocytes $\left(\times 10^{5} / \mathrm{mL}\right)$ & $0.40(0.26-0.62)$ & $0.35(0.20-0.80)$ & 0.45 \\
\hline
\end{tabular}

aspirin did not impede neutrophil phagocytosis (see online supplementary data figure S6).

\section{Aspirin reduces BAL and systemic thromboxane B2 levels}

Consistent with its known effect of circulating platelets, volunteers in the aspirin-treated groups had reduced plasma concentrations of thromboxane B2 (figure 6A). Interestingly, aspirin treatment was also associated with a significant reduction in BAL thromboxane B2 concentrations also (figure 6B).

\section{High-dose versus low-dose aspirin in the human healthy volunteer model}

The a priori statistical analysis plan was to combine data from the aspirin-treated cohorts and compare with placebo. A secondary analysis of high-dose versus low-dose aspirin showed no consistent difference between the two groups (table 4). For neutrophil-derived enzymes, MPO was lower in the high-dose aspirin group, while NE was lower in the $75 \mathrm{mg}$ aspirin group.

\section{Aspirin reduces BAL neutrophils and histological injury score} in the ex vivo perfused and injured lung

Consistent with its effects in the healthy volunteer model of LPS inhalation, aspirin reduced BAL neutrophilia in human lungs injured with LPS ex vivo (figure 7A) and reduced evidence of lung injury as assessed at histological examination (figure $7 \mathrm{~B}, \mathrm{C}$ ). Aspirin did not improve alveolar fluid clearance (AFC), a marker of intact epithelial function, in the ex vivo injured lung (not shown).

\section{DISCUSSION}

While prior preclinical and observational studies have indicated aspirin may have beneficial effects in ARDS, these are the first prospective data indicating a beneficial effect on alveolar inflammation in human subjects, and the first data showing aspirin reduces histological alveolar injury in human lungs.

Aspirin did not significantly reduce the prespecified primary outcome measure of BAL IL- 8 concentration. In this study, the SD of BAL IL-8 in healthy donors after LPS inhalation was higher than we have found in a previous study and the study was therefore underpowered for this outcome measure. Interestingly while aspirin did not significantly reduce IL-8, it did reduce neutrophil infiltration to the alveolar space in both the healthy volunteer study and the EVLP model, suggesting other mediators of neutrophil chemoattraction were affected.

Aspirin reduced BAL concentrations of neutrophil-specific protease MMP-8 as well as MMP-9. Both of these proteases are
A

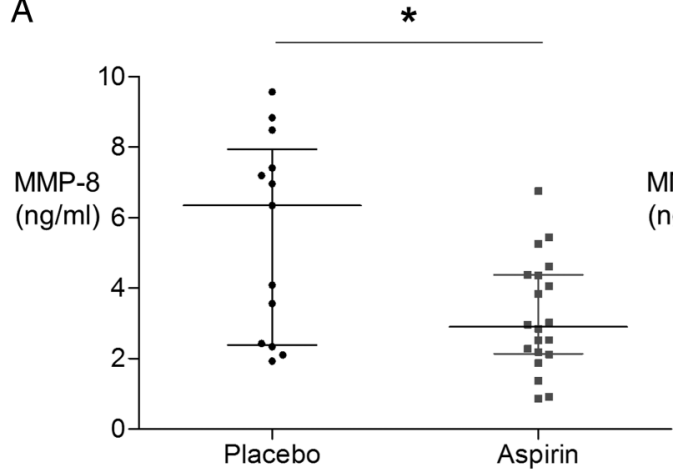

C

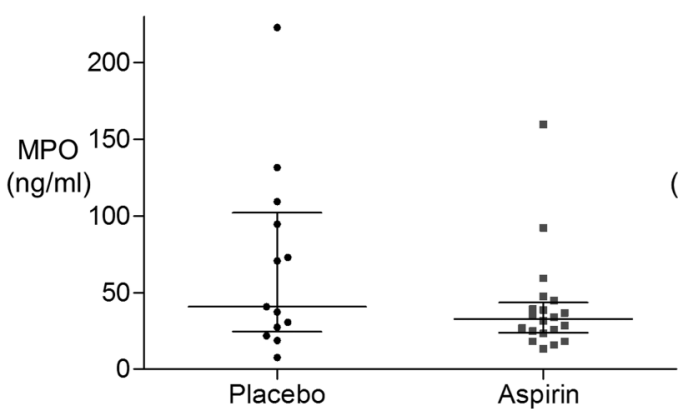

B

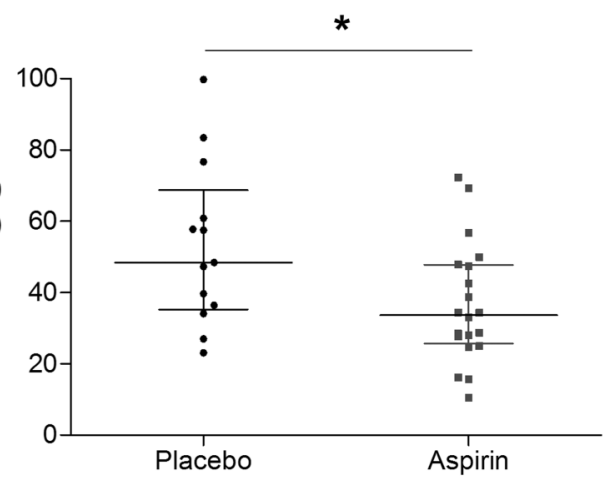

$\mathrm{D}$

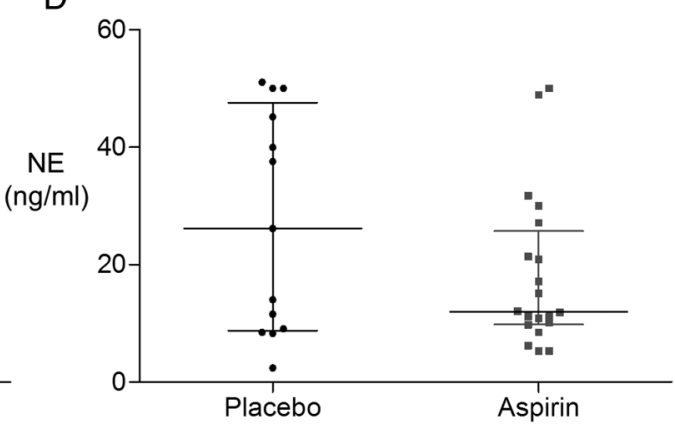

Figure 3 Aspirin reduces neutrophil proteases in the bronchoalveolar lavage (BAL) fluid of healthy volunteers who have inhaled lipopolysaccharide (LPS). BAL as in figure 2A was analysed for neutrophil granular enzymes. (a) Aspirin significantly reduced BAL MMP-8 (difference in median $2.2 \mathrm{ng} /$ $\mathrm{mL}, 95 \% \mathrm{Cls} 0.15$ to $4.45 ;{ }^{*} \mathrm{p}=0.04$ for aspirin $(\mathrm{n}=20)$ vs. placebo $(\mathrm{n}=13)$ ). (B) Aspirin significantly reduced BAL MMP-9 (difference in medians

$14.2 \mathrm{ng} / \mathrm{mL}, 95 \%$ Cls 1.0 to $29.8 ;{ }^{*} p=0.04$ for aspirin $(n=20)$ vs. placebo $(n=13)$ ). (C) Aspirin did not significantly reduce MPO ( $\left.n=20\right)$ versus control $(n=13)$ (difference in median $12.4 \mathrm{ng} / \mathrm{mL}, 95 \%$ Cls -6.6 to $52.6 ; p=0.19)$. (D) Aspirin $(n=20)$ did not statistically significantly reduce BAL neutrophil elastase compared with placebo $(n=13)$ (median difference $4.5 \mathrm{ng} / \mathrm{mL}, 95 \% \mathrm{Cls}-3.0$ to $26.4 ; \mathrm{p}=0.30$ ). 
Table 3 The effect of aspirin on bronchoalveolar lavage (BAL) cytokines in healthy volunteers who inhaled lipopolysaccharide

\begin{tabular}{|c|c|c|c|c|}
\hline BAL analyte & Placebo & Aspirin & Difference in median $(95 \% \mathrm{Cl})$ & p Value \\
\hline $\mathrm{TNF} \alpha(\mathrm{pg} / \mathrm{mL})$ & $106(87-154)$ & $78(52-128)$ & 33 (1.91 to 63.74$)$ & 0.03 \\
\hline IL-1 $\beta$ (pg/mL) & 43 (26-99) & $37(19-55)$ & $13(-6$ to 46$)$ & 0.17 \\
\hline IL-1 $\beta: I L-1 R a$ & $0.02(0.01-0.03)$ & $0.01(0.008-0.014)$ & $0.01(-.0 .001$ to 0.02$)$ & 0.07 \\
\hline IL-6 (pg/mL) & 857 (606-1263) & $648(330-835)$ & $258(-40$ to 526$)$ & 0.07 \\
\hline IL-8 (pg/mL) & $448(321-657)$ & $346(275-508)$ & $102(-40$ to 253$)$ & 0.11 \\
\hline MCP-1 (pg/mL) & $547(412-1055)$ & $502(348-888)$ & 83 (-168 to 363$)$ & 0.45 \\
\hline CRP (pg/mL) & $332(93-1140)$ & 308 (114-824) & 9 (-351 to 266$)$ & 0.99 \\
\hline
\end{tabular}

IL, interleukin; TNF $\alpha$, tumour necrosis factor $\alpha$; MCP, Monocyte chemoattractant protein.
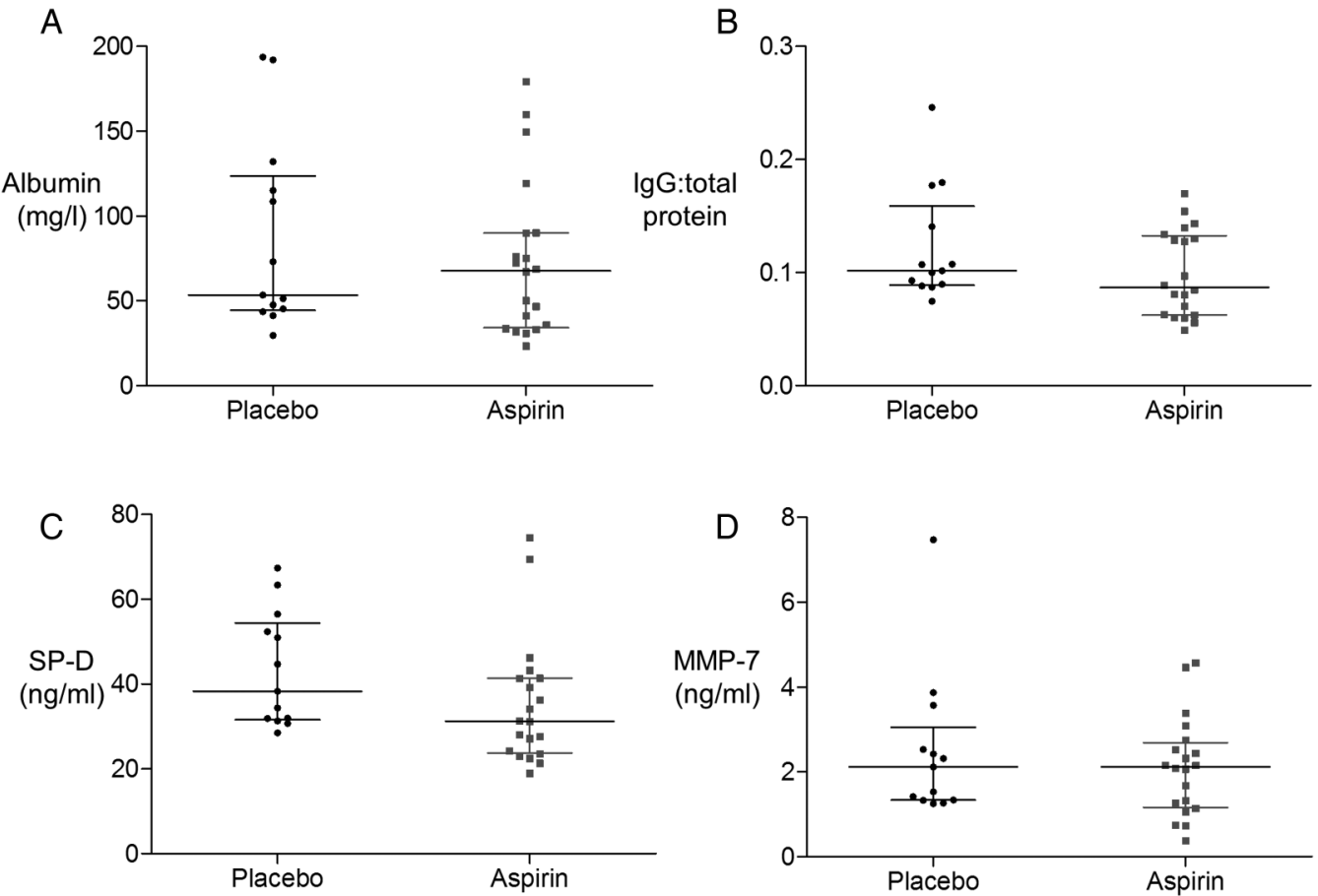

Figure 4 Aspirin does not reduce alveolar leak nor markers of epithelial injury/activation in bronchoalveolar lavage (BAL) in healthy volunteers who have inhaled lipopolysaccharide. BAL as in figure 2 was analysed for (A) albumin as a marker of alveolar barrier integrity and (B) IgG:total protein ratio. Neither was affected by aspirin pretreatment. For albumin, difference in medians was $9.7 \mathrm{mg} / \mathrm{L}, 95 \% \mathrm{Cls}-23.5$ to 41.2 . For $\mathrm{lgG}$ :total, protein difference in medians was $0.02,95 \% \mathrm{Cls}-0.01$ to 0.04 . (C) Aspirin did not affect BAL SP-D, a marker of type II alveolar epithelial cell activation; difference in medians $8.4 \mathrm{ng} / \mathrm{mL}, 95 \% \mathrm{Cls}-0.5$ to 19.5 . (D) Aspirin did not reduce BAL MMP-7, an epithelial cell-derived protease in the alveolar space; difference in medians $0.2 \mathrm{ng} / \mathrm{mL}, 95 \% \mathrm{Cls}-0.7$ to 1.0 ). $\mathrm{n}=13$ for placebo, $\mathrm{n}=20$ for aspirin for all measures.

present in preformed states within neutrophils, in the secondary (MMP-8) and tertiary granules (MMP-9). While MMP-9 is also secreted by other cells within the alveolus, including epithelial cells and macrophages, ${ }^{26}$ the neutrophil is recognised to be a major source of its production in ARDS. ${ }^{27}$ The reduction in these proteases implies that in addition to reduced overall numbers of neutrophils in the alveolar space in the aspirintreated group, there are less degranulated neutrophils here also. A reduction in MMP-9 and MMP-8 may reduce tissue injury in the early stages of alveolar injury.

LPS inhalation drives activation of TLR-4 and downstream activation of nuclear factor $\kappa \mathrm{B}(\mathrm{NF} \kappa \mathrm{B})$-dependent cytokine production. Aspirin significantly reduced secretion of the pro-inflammatory cytokine TNF $\alpha$. Macrophage-derived TNF $\alpha$ has long been identified as a key factor driving activation of neutrophils in ARDS. ${ }^{28}$ In vivo models show that selective inhibition of the pro-inflammatory signalling TNFR1 (p55) reduces neutrophil recruitment and injury in acid-and ventilator-induced lung ARDS. ${ }^{29} \mathrm{~A}$ trend to reduction in secretion of the other major pro-inflammatory cytokines implicated in ARDS (including IL- 6 and IL1 $\beta$ net activity as assessed by IL-1 $\beta$ :IL-1Ra ratio) was seen but did not reach statistical significance. Aspirin in experimental studies has been shown to

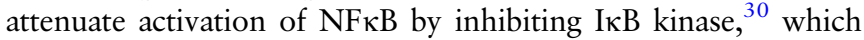
may be a potential mechanism of reduction in the secretion of pro-inflammatory cytokines seen in this study.

Importantly, although aspirin had a clear effect on neutrophilic inflammation in this model it did not have any obvious effect on epithelial barrier function, as assessed by protein leak. Altered neutrophil infiltration to the alveolus in response to injury without changes in epithelial barrier function has been reported by other investigators, ${ }^{31} 32$ suggesting that the two processes, both pathophysiological hallmarks of ARDS, may be differentially regulated. SP-D is reduced in the alveolar space in ARDS,${ }^{33}$ and in our study aspirin had no effect on this type II epithelial cell marker implying aspirin does not exert its effect 
A

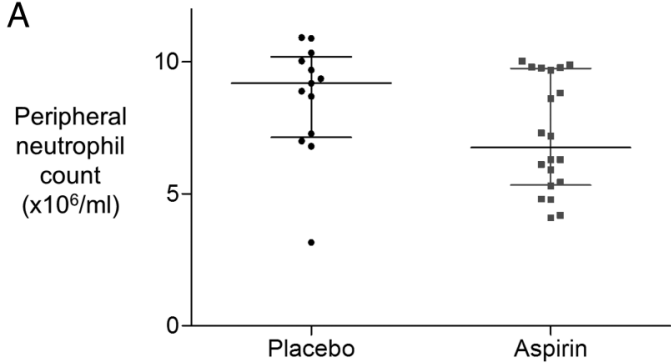

B

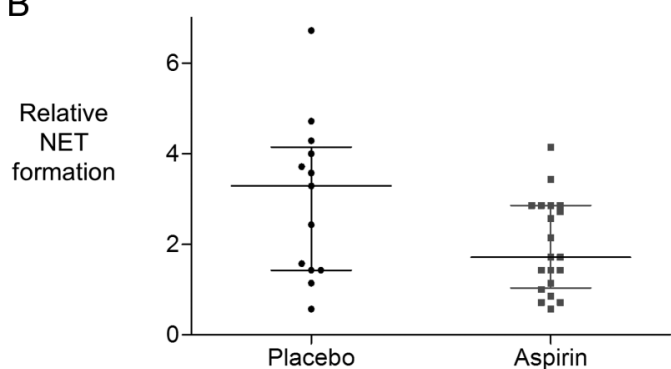

Figure 5 The effect of aspirin on peripheral neutrophil count. (A) Peripheral neutrophil count in healthy volunteers 6 hours after lipopolysaccharide (LPS) inhalation is not significantly reduced by aspirin, difference in medians $1.47 \times 106 / \mathrm{mL}, 95 \% \mathrm{Cls}-0.1$ to 3.4 , $p=0.07$. (B) Neutrophil extracellular trap (NET) formation in blood 6 hours after LPS inhalation was not statistically significantly reduced in the aspirin-treated cohort $(n=20)$ versus placebo $(n=13)$, difference in medians $0.9,95 \% \mathrm{Cls}-0.1$ to 2,$1 ; p=0.09$. Data are expressed as multiples of mean optical density (OD) values obtained for NETs in normal blood.

A

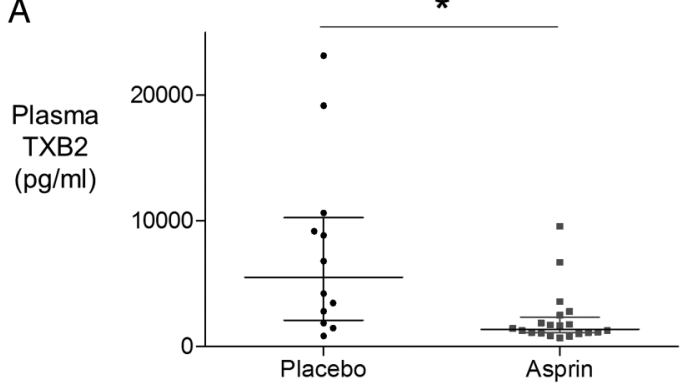

B

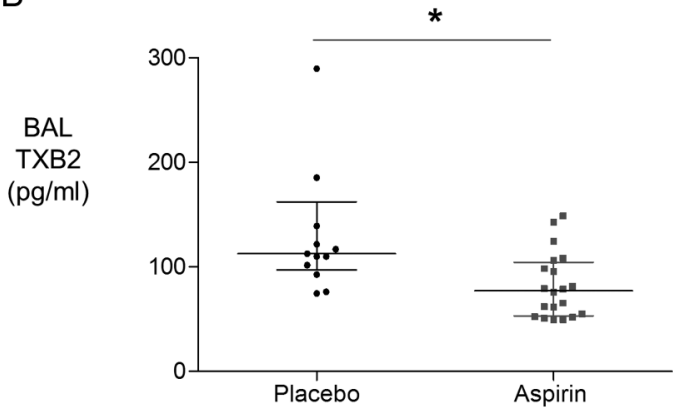

Figure 6 The effect of aspirin on bronchoalveolar lavage (BAL) and plasma thromboxane B2 (TXB2) after lipopolysaccharide inhalation (A) Plasma thromboxane B2 was reduced by aspirin treatment $(n=12$ placebo, $\mathrm{n}=20$ aspirin). Difference in medians $3077 \mathrm{pg} / \mathrm{mL}, 95 \% \mathrm{Cls} 755$ to 7850 ; ${ }^{*} p=0.003$. (B) BAL thromboxane B2 was significantly reduced in the aspirin-treated cohort $(n=20)$ compared with placebo $(n=13)$. One outlier from the placebo group is not shown within the range of the graph (value $>7000 \mathrm{pg} / \mathrm{mL}$ ), but is included in statistical analysis presented. Difference in medians $41 \mathrm{pg} / \mathrm{mL}, 95 \%$ Cls 14.7 to 64.3 ; ${ }^{*} \mathrm{p}=0.003$. (BAL TXB2 is also significantly reduced in response to aspirin with this outlier excluded.)
Table 4 The effect of high-dose versus low-dose aspirin on bronchoalveolar lavage (BAL) and plasma biomarkers

\begin{tabular}{|c|c|c|}
\hline & Aspirin 75 mg & Aspirin $1200 \mathrm{mg}$ \\
\hline \multicolumn{3}{|l|}{ BAL } \\
\hline Neutrophils $\left(\times 10^{5} / \mathrm{mL}\right)$ & $1.6(1.3-2.8)$ & $1.5(1.2-1.7)$ \\
\hline MMP-8 (ng/mL) & $3.4(2.3-4.8)$ & $2.6(1.9-4.0)$ \\
\hline MMP-9 (ng/mL) & $33.7(27.1-50.1)$ & 33.7 (16.1-44.4) \\
\hline MPO (ng/mL) & $38.6(29.2-53.3)$ & $25.2(17.5-34.6)$ \\
\hline $\mathrm{NE}(\mathrm{ng} / \mathrm{mL})$ & $10.6(6.0-15.6)$ & $21.1(11.1-36.1)$ \\
\hline $\mathrm{TNF} \alpha(\mathrm{pg} / \mathrm{mL})$ & $77(51-100)$ & $90(49-141)$ \\
\hline IL-6 (pg/mL) & $563(368-880)$ & 782 (284-901) \\
\hline IL-8 (pg/mL) & $308(264-431)$ & $397(269-515)$ \\
\hline MCP-1 (pg/mL) & $497(385-967)$ & $502(289-924)$ \\
\hline $\mathrm{IL}-1 \beta(p g / m L)$ & $46(30-66)$ & $30(16-38)$ \\
\hline IL-1Ra (pg/mL) & $3000(2413-4182)$ & $2266(1700-3682)$ \\
\hline TIMP-1 (pg/mL) & 2155 (1431-3783) & 2243 (1275-3789) \\
\hline TIMP-2 (pg/mL) & 566 (495-912) & $574(224-805)$ \\
\hline SP-D (pg/mL) & $27810(23$ 356-38 705) & 35242 (23 800-41 848) \\
\hline CRP (pg/mL) & 449 (97-943) & 237 (116-867) \\
\hline \multicolumn{3}{|l|}{ Plasma } \\
\hline CRP (mg/L) & $40(33-61)$ & $32(25-63)$ \\
\hline
\end{tabular}

directly on this epithelial cell. In addition, the absence of an effect on MMP-7, the most abundant source of which in the lung is the epithelium, ${ }^{34}$ also suggests aspirin has little direct effect on the epithelium. Taken together the data suggest aspirin may have a significantly anti-inflammatory effect during the early injury phase of ARDS, without a direct effect on protein leak.

The ex vivo lung perfusion model has been used in previous studies as a human model of ARDS. ${ }^{18}$ LPS administered intrabronchially resulted in a significant increase in infiltration of neutrophils from the blood added to the perfusate into the alveoli. Neutrophil sequestration in the alveoli characterises ARDS. ${ }^{35}$ In addition to replicating the findings of reduced neutrophil recruitment to the alveolar space in the aspirin-treated group as seen in the healthy volunteer model, we were able to assess the effect of aspirin on whole tissue inflammation by histology. The scoring system used was a modification of that proposed by Matute-Bello et al. ${ }^{25}$ Normal alveolar structure is characterised by thin alveolar walls with macrophages and neutrophils being rarely present, while in ARDS injured lungs show increased alveolar septal thickness, intra-alveolar haemorrhage, protein debris and neutrophilia. Pretreatment with aspirin significantly reduced the severity of injury in this model and likely reflects reduced neutrophil-dependent injury. Similar to the human volunteer model, aspirin had no effect on epithelial function as measured by AFC.

Systemically a trend to reduction in neutrophil count occurred but did not reach statistical significance. Importantly, aspirin did not impair neutrophil phagocytic uptake of opsonised zymosan beads in vitro, implying aspirin may be used for antiinflammatory effects while retaining the capacity to engulf pathogens. We recognise this assay did not measure aspirin's effect on phagocytosis in vivo, which may differ from its in vitro activity.

Recent studies have highlighted the importance of plateletneutrophil interaction in NETs formation. NETs have been shown to have bactericidal properties, which damage native 
A

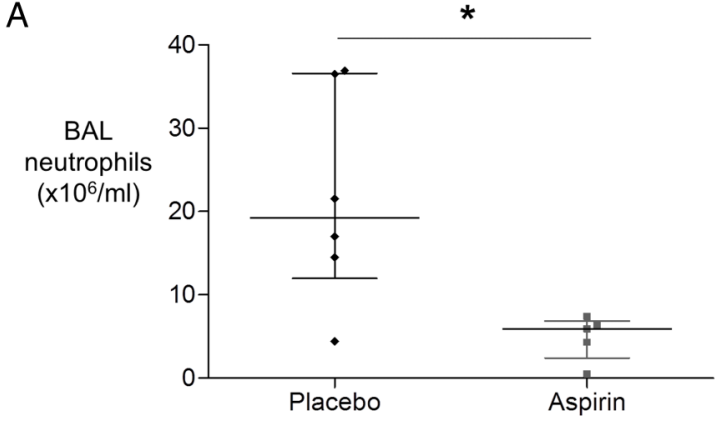

B

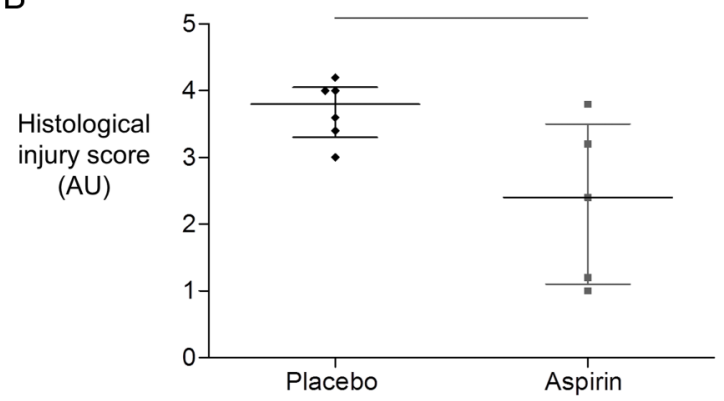

C
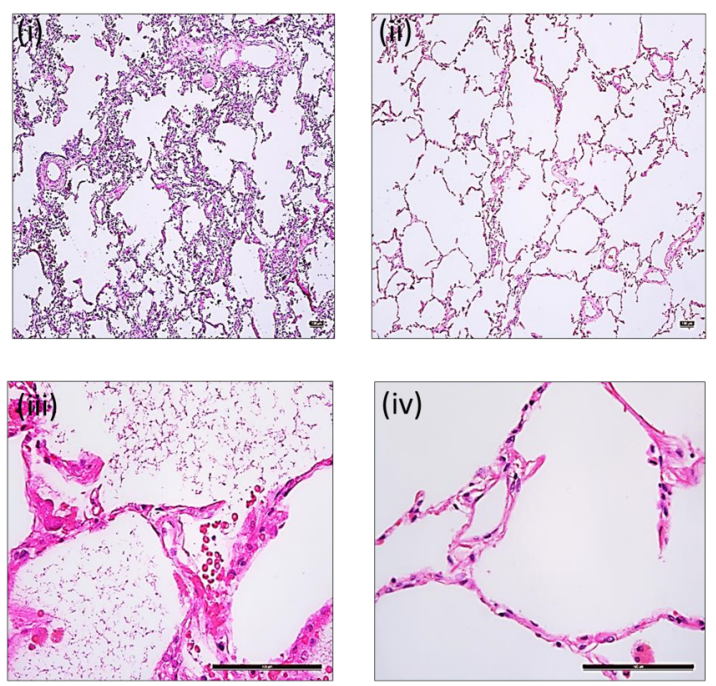

Figure 7 Aspirin significantly reduces neutrophilic inflammation in the human ex vivo lung perfusion model. Human lungs were ventilated and perfused ex vivo. Blood was preincubated with aspirin $(n=5)$ or placebo $(n=6)$ for 1 hour before adding to the lung perfusate and simultaneously injuring the lung with lipopolysaccharide (LPS) $(6 \mathrm{mg})$. Bronchoalveolar lavage (BAL) was carried out 4 hours after injury. (A) Aspirin significantly reduces BAL neutrophilia. Neutrophils in BAL fluid were counted in cytospins. ${ }^{*} p=0.03$ for aspirin $(n=5)$ versus placebo $(n=6), M W U$ test. (B) Ventilated perfused isolated human lungs that were injured ex vivo with LPS had reduced injury as measured by lung injury score when treated with aspirin $(n=5)$ versus placebo $(n=6)$. y axis, AU , arbitrary units), ${ }^{*} p=0.05 \mathrm{MWU}$ test. (C) Illustrative histological sections of lung are shown. (i) and (iii) are placebo-treated lungs at low and high power, respectively; (ii) and (iv) are aspirin-treated lungs at low and high power. Scale bar represents $100 \mu \mathrm{m}$. Alveolar septal wall thickening, neutrophil recruitment and proteinaceous debris staining are less marked in the aspirin than the placebo-treated group. Quantification of lung injury score was carried out in a blinded manner to counter any potential bias in image selection. cells ${ }^{36}$ and have also been implicated in the pathology of ARDS. Platelet-dependent NETs formation drove pulmonary platelet sequestration, capillary permeability and pulmonary oedema in an in vivo model of transfusion-related acute lung injury. ${ }^{7}$ In our study, median plasma NETs formation fell by approximately $30 \%$ in the aspirin-treated cohort, but did not reach statistical significance. It is possible that reduced plasma NETs may have contributed to the reduced neutrophil influx into the alveolar space.

LPA formation has been implicated in driving pulmonary and systemic inflammation, ${ }^{3}$ and although both monocyte-platelet and neutrophil-platelet aggregates were demonstrable in this study, aspirin did not reduce their formation, unlike in the case of LPS challenge in mice. ${ }^{5}$ It is possible that in LPS-stimulated inflammation in humans, similar to patients with atherosclerosis, ${ }^{37}$ aspirin does not reduce the platelet surface expression of p-selectin sufficiently to inhibit LPA formation.

Inhibition of COX-1 in platelets leads to reduced thromboxane A secretion. Thromboxane A is rapidly metabolised to its more stable metabolite thromboxane B2. ${ }^{38}$ We demonstrated a reduction in thromboxane $\mathrm{B} 2$ in the plasma of patients taking aspirin, confirming that the medication had been taken, and the cohort was not 'aspirin-resistant'. ${ }^{39}$ Reduction in systemic thromboxanes may be related to the trend to reduction in NETs formation in the aspirin-treated cohort since NETs formation is thromboxane-dependent. ${ }^{7}$

Interestingly, we demonstrated reduced thromboxane $\mathrm{B} 2$ in the BAL of the aspirin-treated volunteers, consistent with reduced platelet activation in the lung. Although thromboxanes can be produced by cells other than platelets, ${ }^{40}$ the quantities are much lower. The high concentrations measured in this study, given an approximately 100 -fold dilutional effect of BAL, lead us to speculate that LPS inhalation may drive platelet activation within the human alveolar compartment, as has been shown in the murine lung. ${ }^{5}$

This is the first human study to compare two different doses of aspirin in the setting of pulmonary inflammation. The rationale for this was to assess whether low-dose aspirin (that primarily has an antiplatelet effect) or high-dose (that has a direct anti-inflammatory effect by reducing $\mathrm{NF \kappa B}$-dependent gene transcription in tissues) was effective in reducing pulmonary injury induced by LPS inhalation. There was no dose-dependent effect within the aspirin subgroups in the clinical study. The lower dose is sufficient to inhibit platelet activation and thromboxane release. Given that this was associated with reduced NETs and pulmonary neutrophilia, we speculate that aspirin inhibits neutrophilic inflammation in a platelet-dependent manner potentially via reduced thromboxane-dependent NETs formation required for neutrophil migration to the lung. Also, $1200 \mathrm{mg}$ aspirin was used as the higher dose and is associated with anti-inflammatory effects in humans, but was not additional in this model. Higher doses of up to $8 \mathrm{~g} /$ day have been used in rheumatic diseases for their anti-inflammatory effect, but are associated with increased gastrointestinal side effects and salicylism. It is possible that a higher dose of aspirin in this model would have shown additional anti-inflammatory activity but we felt was unlikely to be clinically appropriate for critically ill patients. Given that low-dose aspirin is less likely to cause adverse effects in the critically ill patient population and shows efficacy in this model, future clinical trials testing aspirin in the 
prevention or treatment of ARDS should be designed using lower-dose aspirin.

We acknowledge that our models have limitations. In the healthy volunteer model of LPS inhalation, the nature of injury is mild and self-limiting compared with ARDS. BAL at a single time point does not capture the dynamic changes that take place in the inflamed alveoli in ARDS. The healthy participants lack comorbidities and poly-pharmacy. This was a pretreatment study designed to test the proof of concept that aspirin could reduce pulmonary inflammation. Due to the short-term nature of the model, a postinjury treatment would not have allowed us to test the effect of aspirin, particularly low-dose aspirin, as it takes $>6$ hours for $75 \mathrm{mg}$ aspirin to achieve full platelet inhibition, and further downstream effects. Pretreatment is potentially relevant for those who can be identified as being at high risk of developing ARDS, and pre-existing aspirin therapy may have protective pulmonary effects for those undergoing surgery that predisposes to ARDS, for example, coronary artery bypass grafting or abdominal aortic aneurysm repair. Our model does not address whether aspirin withdrawal in the perioperative period increases the risk of pulmonary inflammation, and most studies looking at the effect of aspirin withdrawal perioperatively have focused on cardiovascular and bleeding outcomes rather than pulmonary complications. ${ }^{41}{ }^{42}$ Although the EVLP model allows a greater induction of injury by using a higher LPS dose, it has a short life, preventing use of these lungs to study fibrotic or resolution phases of ARDS. The EVLP model lacks hepatorenal metabolism and a reticuloendothelial system, which may modulate the drug and inflammatory response, respectively. Additionally, the lungs have undergone a period of cold ischaemia and had been rejected for transplantation so there is potential variability in the baseline degree of injury. We try to minimise this by using only lungs that have intact fluid clearance at baseline and no gross evidence of haemorrhage, contusion or consolidation. Furthermore, the underlying mechanisms leading to ICU admission and brain death, and the treatments the donor may have received in ICU may lead to altered response to either LPS or aspirin. However, again we aimed to minimise any confounding from this by randomisation and blinding. In the EVLP model, the blood volume is diluted (1/10), which has the potential to affect rheology, intercellular interaction and cell recruitment to the lung. Unfortunately, it is not possible to obtain $1 \mathrm{~L}$ of blood from a single donor for research purposes to avoid this. In both models, the injury LPS is a bacterial antigen, rather than a live bacterium, and isolated TLR4 stimulation does not recapitulate the myriad of stimuli that drive ARDS in patients.

Nevertheless, both models have the advantage of being carried out in human tissues, proving prospectively that aspirin at clinically relevant doses can attenuate neutrophilic human alveolar inflammation, and histological alveolar injury, but does not affect epithelial barrier function. These data support the further testing of aspirin in clinical trials as an anti-inflammatory strategy for treatment of neutrophilic-driven pulmonary inflammatory diseases such as ARDS.

\footnotetext{
Author affiliations

${ }^{1}$ Centre for Experimental Medicine, School of Medicine, Dentistry and Biomedical Sciences, Queen's University Belfast, Belfast, Northern Ireland, UK

${ }^{2} S c h o o l$ of Pharmacy, Queen's University of Belfast, Belfast, Northern Ireland, UK ${ }^{3}$ Department of Medicine, University of California San Francisco, San Francisco, California, USA

${ }^{4}$ Northern Ireland Clinical Trials Unit, Royal Victoria Hospital, Belfast, UK ${ }^{5}$ Institute of Cellular Medicine, Medical School, Newcastle University, Newcastleupon Tyne, UK
}

${ }^{6}$ Regional Intensive Care Unit, Royal Victoria Hospital, Belfast Health and Social Care Trust, Belfast, UK

Acknowledgements The authors thank the volunteers who participated in the study, the endoscopy staff and the Northern Ireland Clinical Research Facility at Belfast City Hospital, Ms C McDowell from the Northern Ireland CTU for statistical advice, and the funders (Intensive Care Society, UK, and Intensive Care Society of Ireland).

Contributors UH contributed to clinical study conduct, the ex vivo experiments, laboratory analyses, data analysis and manuscript preparation. MF contributed to ex vivo experiments, laboratory analyses, data analysis and manuscript preparation. MS contributed to study design, obtaining funding, clinical study, data analysis and manuscript preparation. RV contributed to study design, data analysis. AK, CS, EL, MRL, JS and AJS contributed to laboratory analyses, data analysis and manuscript preparation. JMCN contributed to study design, lab analysis, data analysis and manuscript preparation. DFMcA contributed to study design, obtaining funding, clinical study, ex vivo experiments, data analysis and manuscript preparation. CMO contributed to study design, obtaining funding, clinical study, ex vivo experiments, lab analyses, data analysis and manuscript preparation.

Funding The UK Intensive Care Society and the Intensive Care Society of Ireland. Competing interests None declared.

Patient consent Obtained.

Ethics approval ORECNI for clinical study, SREC at QUB for the EVLP study.

Provenance and peer review Not commissioned; externally peer reviewed.

\section{REFERENCES}

1 Blair P, Flaumenhaft R. Platelet alpha-granules: basic biology and clinical correlates. Blood Rev 2009;23:177-89.

2 Grommes J, Alard JE, Drechsler M, et al. Disruption of platelet-derived chemokine heteromers prevents neutrophil extravasation in acute lung injury. Am J Respir Crit Care Med 2012;185:628-36.

3 Sreeramkumar V, Adrover JM, Ballesteros I, et al. Neutrophils scan for activated platelets to initiate inflammation. Science (New York, NY) 2014;346:1234-8.

4 Zarbock A, Singbartl K, Ley K. Complete reversal of acid-induced acute lung injury by blocking of platelet-neutrophil aggregation. J Clin Invest 116:3211-19.

5 Ortiz-Muñoz G, Mallavia B, Bins A, et al. Aspirin-triggered 15-epi-lipoxin A4 regulates neutrophil-platelet aggregation and attenuates acute lung injury in mice. Blood 2014;124:2625-34.

6 Clark SR, Ma AC, Tavener SA, et al. Platelet TLR4 activates neutrophil extracellular traps to ensnare bacteria in septic blood. Nat Med 2007;13:463-9.

7 Caudrillier A, Kessenbrock K, Gilliss BM, et al. Platelets induce neutrophil extracellular traps in transfusion-related acute lung injury. J Clin Invest 2012;122:2661-71.

8 Narasaraju T, Yang E, Samy RP, et al. Excessive neutrophils and neutrophil extracellular traps contribute to acute lung injury of influenza pneumonitis. Am J Pathol 2011;179:199-210.

9 Sayah DM, Mallavia B, Liu F, et al. Neutrophil extracellular traps are pathogenic in primary graft dysfunction after lung transplantation. Am J Respir Crit Care Med 2015;191:455-63.

10 Serhan CN, Chiang N, Van Dyke TE. Resolving inflammation: dual anti-inflammatory and pro-resolution lipid mediators. Nat Rev Immunol 2008;8:349-61.

11 Looney MR, Nguyen JX, Hu Y, et al. Platelet depletion and aspirin treatment protect mice in a two-event model of transfusion-related acute lung injury. J Clin Invest 2009;119:3450-61.

12 Erlich JM, Talmor DS, Cartin-Ceba R, et al. Prehospitalization antiplatelet therapy is associated with a reduced incidence of acute lung injury: a population-based cohort study. Chest 2011;139:289-95.

13 O'Neal HR, Koyama T, Koehler EAS, et al. Prehospital statin and aspirin use and the prevalence of severe sepsis and ALI/ARDS. Crit Care Med 2011;39:1343-50.

14 Harr JN, Moore EE, Johnson J, et al. Anti-platelet therapy is associated with decreased transfusion-associated risk of lung dysfunction, multiple organ failure, and mortality in trauma patients. Crit Care Med 2013;41:399-404.

15 Kor DJ, Carter RE, Park PK, et al. Effect of aspirin on development of ards in at-risk patients presenting to the emergency department: the LIPS-a randomized clinical trial. JAMA 2016;315:2406-14

16 Boyle AJ, Di Gangi S, Hamid UI, et al. Aspirin therapy in patients with acute respiratory distress syndrome (ARDS) is associated with reduced intensive care unit mortality: a prospective analysis. Crit Care 2015;19:109.

17 Shyamsundar M, McKeown STW, O'Kane CM, et al. Simvastatin decreases lipopolysaccharide-induced pulmonary inflammation in healthy volunteers. Am J Respir Crit Care Med 2009;179:1107-14.

18 Lee JW, Fang X, Gupta N, et al. Allogeneic human mesenchymal stem cells for treatment of $E$. coli endotoxin-induced acute lung injury in the ex vivo perfused human lung. Proc Natl Acad Sci USA 2009;106:16357-62 
19 Spence $S$, Greene MK, Fay F, et al. Targeting Siglecs with a sialic acid-decorated nanoparticle abrogates inflammation. Sci Trans/ Med 2015;7:303ra140.

20 McAuley DF, Curley GF, Hamid UI, et al. Clinical grade allogeneic human mesenchymal stem cells restore alveolar fluid clearance in human lungs rejected for transplantation. Am J Physiol 2014;306:L809-15.

21 Shyamsundar M, McAuley DF, Ingram RJ, et al. Keratinocyte growth factor promotes epithelial survival and resolution in a human model of lung injury. Am J Respir Crit Care Med 2014;189:1520-9.

22 Goldstein SL, Leung JC, Silverstein DM. Pro- and anti-inflammatory cytokines in chronic pediatric dialysis patients: effect of aspirin. Clin J Am Soc Nephrol 2006:1:979-86.

23 Seymour RA, Rawlins MD. Efficacy and pharmacokinetics of aspirin in post-operative dental pain. Br I Clin Pharmacol 1982;13:807-10.

24 Morris AC, Kefala K, Wilkinson TS, et al. C5a mediates peripheral blood neutrophil dysfunction in critically III patients. Am J Respir Crit Care Med 2009;180:19-28.

25 Matute-Bello G, Downey G, Moore BB, et al. An Official American Thoracic Society Workshop Report: features and measurements of experimental acute lung injury in animals. Am J Respir Cell Mol Biol 2011;44:725-38.

26 O'Kane CM, McKeown SW, Perkins GD, et al. Salbutamol up-regulates matrix metalloproteinase-9 in the alveolar space in the acute respiratory distress syndrome. Crit Care Med 2009:37:2242-9.

27 Steinberg J, Halter J, Schiller HJ, et al. Metalloproteinase inhibition reduces lung injury and improves survival after cecal ligation and puncture in rats. J Surg Res 2003:111:185-95

28 Larrick JW, Graham D, Toy K, et al. Recombinant tumor necrosis factor causes activation of human granulocytes. Blood 1987:69:640-4.

29 Bertok S, Wilson MR, Morley PJ, et al. Selective inhibition of intra-alveolar p55 TNF receptor attenuates ventilator-induced lung injury. Thorax 2012:67:244-51.

30 Yoo CG, Lee S, Lee CT, et al. Effect of acetylsalicylic acid on endogenous I kappa B kinase activity in lung epithelial cells. Am J Physiol Lung Cell Mol Physiol 2001;280: L3-9.
31 Martin TR, Pistorese BP, Chi EY, et al. Effects of leukotriene B4 in the human lung. Recruitment of neutrophils into the alveolar spaces without a change in protein permeability. I Clin Invest 1989;84:1609-19.

32 Wiener-Kronish JP, Albertine KH, Matthay MA. Differential responses of the endothelial and epithelial barriers of the lung in sheep to Escherichia coli endotoxin. J Clin Invest 1991;88:864-75.

33 Cheng IW, Ware LB, Greene KE, et al. Prognostic value of surfactant proteins $A$ and D in patients with acute lung injury. Crit Care Med 2003:31:20-7.

34 Dunsmore SE, Saarialho-Kere UK, Roby JD, et al. Matrilysin expression and function in airway epithelium. J Clin Invest 1998;102:1321-31.

35 Weiland JE, Davis WB, Holter JF, et al. Lung neutrophils in the adult respiratory distress syndrome. Clinical and pathophysiologic significance. Am Rev Respir Dis 1986:133:218-25.

36 Brinkmann V, Reichard U, Goosmann C, et al. Neutrophil extracellular traps kill bacteria. Science 2004:303:1532-5.

37 Klinkhardt U, Bauersachs R, Adams J, et al. Clopidogrel but not aspirin reduces P-selectin expression and formation of platelet-leukocyte aggregates in patients with atherosclerotic vascular disease*. Clin Pharmacol Ther 2003;73: 232-41.

38 Catella F, Healy D, Lawson JA, et al. 11-Dehydrothromboxane B2: a quantitative index of thromboxane A2 formation in the human circulation. Proc Natl Acad Sci USA 1986;83:5861-5.

39 Hankey GJ, Eikelboom JW. Aspirin resistance. Lancet 367:606-17.

40 Zheng $\mathrm{H}$, Crowley JJ, Chan JC, et al. Attenuation of LPS-induced neutrophil thromboxane b2 release and chemiluminescence. J Cell Physiol 1991;146: 264-9.

41 Devereaux PJ, Mrkobrada M, Sessler DI, et al. Aspirin in patients undergoing noncardiac surgery. New Engl J Med 2014;370:1494-503.

42 Gerstein NS, Schulman PM, Gerstein WH, et al. Should more patients continue aspirin therapy peri-operatively?: clinical impact of aspirin withdrawal syndrome. Ann Surg 2012;255:811-19. 